\title{
Application of aerodynamic model structure determination to UAV data
}

\author{
S. Carnduff \\ sdcarnduff@yahoo.co.uk \\ A. Cooke \\ Department of Aerospace Sciences \\ Cranfield University \\ Cranfield, UK
}

\begin{abstract}
This paper concerns aircraft system identification and, in particular, the process of aerodynamic model structure determination. Its application to experimental data from unmanned aerial vehicles (UAVs) is also described. The procedure can be particularly useful for determining an aerodynamic model for aircraft with unconventional airframe configurations, which some unmanned aircraft tend to have. Two model structure determination techniques are outlined. The first is the well-established stepwise regression method, while the second is an adaptation of an existing frequency response approach which instead utilises maximum likelihood estimation. Example applications of the methods are presented for two data sources. The first is a set of UAV flight test data and the second is data recorded from dynamic wind tunnel tests on a UAV configuration. For both examples, the model structures determined using stepwise regression and maximum likelihood analysis matched one another, suggesting that the maximum likelihood approach and the chosen thresholds for its statistical metrics were reliable for the data being analysed.
\end{abstract}

\section{NOMENCLATURE}

$\mathrm{Cl}$ non-dimensional rolling moment coefficient

$\mathrm{Cl}_{p}$ derivative of rolling moment coefficient with respect to roll rate

$\mathrm{Cl}_{r}$ derivative of rolling moment coefficient with respect to yaw rate

$\mathrm{Cl}_{\beta} \quad$ derivative of rolling moment coefficient with respect to angle-of-sideslip

$\mathrm{Cl}_{\zeta} \quad$ derivative of rolling moment coefficient with respect to rudder deflection

$\mathrm{Cl}_{\xi}$ derivative of rolling moment coefficient with respect to aileron deflection

deg degrees

$F_{\text {in }} \quad$ stepwise regression $F$-distribution threshold

$F_{p} \quad$ stepwise regression $F$-distribution threshold partial $F$-statistic

I parameter insensitivity

$J$ cost function $\mathrm{m}$ M

$n$

$n_{p}$

$n_{\zeta}$

$n_{v}$

$n_{0}$

$N$

$p$

$\mathbf{P}$

$r$

$r_{j z}$

$\mathbf{R}$

$R^{2}$

$\mathrm{rad}$

$\mathrm{s}$

$t$

$v$

V

$\mathbf{X}$

$\mathbf{y}$

$y_{p}$ metres

number of unknown parameters to be estimated

Fisher information matrix

number of regressors

derivative of yawing moment with respect to roll rate

derivative of yawing moment with respect to yaw rate

derivative of yawing moment with respect to lateral velocity

derivative of yawing moment with respect to rudder deflection

number of discrete measurement points

roll rate

parameter covariance matrix

yaw rate

correlation between regressor and dependent variable

measurement noise covariance matrix

coefficient of determination

radians

seconds

standard error, wing semi-span

time

lateral velocity component

true airspeed

matrix of regressors

vector of predicted outputs

derivative of sideforce with respect to roll rate

derivative of sideforce with respect to yaw rate

derivative of sideforce with respect to lateral velocity

derivative of sideforce with respect to rudder deflection

dependent variable vector, measurement vector

angle of sideslip

rudder deflection

confidence ellipsoid

parameter vector

atmospheric density, pair-wise correlation coefficient

equation error variance

time delay in rudder data

matrix of residuals

aileron deflection 


\section{Superscripts}

$E \quad$ empirical derivative estimate

$F \quad$ flight test derivative estimate

$T$ matrix transpose

ratio of flight test derivative estimate to empirical derivative estimate

$\wedge \quad$ estimate, non-dimensional motion variable

time derivative

\subsection{INTRODUCTION}

A critical part in the development of any aircraft is establishing its aerodynamic stability and control characteristics. This information is required, for example, in the design of flight control systems, simulator development and handling qualities assessment. Analysis of experimental data provides aerodynamic models of the highest fidelity and the process of system identification, or parameter identification, is used widely as a means of estimating the aerodynamic derivatives from flight and wind tunnel tests.

Comprehensive treatments of both the theoretical and practical aspects of aircraft system identification can be found in Klein and Morelli $^{(1)}$, Jategaonkar ${ }^{(2)}$ and Tischler and Remple ${ }^{(3)}$. Major steps in the system identification process include the following:

- Selection of instrumentation to provide motion variable data of suitable quality.

- Design of control surface input signals to adequately excite the aircraft dynamics and ensure that enough information is present in the measurements to reliably estimate the aerodynamic derivatives of interest.

- Selection of a suitable parameter estimation method to estimate values for the aerodynamic derivatives.

- Validation of the identified aerodynamic model by comparing it against independent data not used in the identification process.

The success of the identification process also depends on the choice of which aerodynamic derivatives to include in the aerodynamic model and what form the aerodynamic model structure should take. Omission of a significant term from the model will lead to an inaccurate representation of the true aircraft, resulting in a model with poor predictive capability. However, inclusion of too many terms in the model and attempting to estimate a large number of parameters from a limited amount of data is likely to result in estimates that are statistically unreliable.

In many cases, the aerodynamic model structure is well defined and is based on physical understanding of the aircraft dynamics. This is particularly true if the vehicle's configuration is conventional and its motion involves small perturbations from an initial trim condition, in which case a linear model is usually applicable. However, specifying the correct model structure may be more difficult if the aircraft's configuration is unconventional or the dynamics being investigated are nonlinear. In analysing data for such an aircraft, it would be convenient to have a systematic, mathematical approach to help determine which terms to be included in the aerodynamic model, rather than repeating the analysis several times using various model structures on a trial-and-error basis.

Methods of aerodynamic model structure determination utilising various statistical metrics were first investigated in the $1970 \mathrm{~s}^{(4-7)}$. The main interest of this research at the time was to construct aerodynamic models from data recorded at high angles-of-attack or from large amplitude manoeuvres, for which the dynamics are nonlinear and less well-understood. The techniques were also used to find global models which could describe the motion of the aircraft over a range of flight conditions. The approach to model structure determination which has arguably received the most attention is stepwise regression, which was first applied to flight test data by Gupta, Hall et $a l^{(6,7)}$ and developed further through the work of Klein et $a l^{(8,9)}$. For this method, a pool of candidate terms for the model is chosen. These terms are then added to the model (but can also be subsequently removed) based on a number of metrics, including Fstatistics, coefficient of determination and predicted sum of squares. The process is halted when no statistical improvement can be made to the model. Another method for model structure determination was proposed by Milne ${ }^{(10)}$, which utilised the frequency response technique developed by Tischler ${ }^{(3)}$ and has been applied widely to rotorcraft applications. In this approach, all candidate terms are initially included in the model. Parameters are then removed based on their estimated standard deviations, insensitivity and correlation with one another. The final model structure is achieved when all parameter values in the model have a similar level of confidence.

The research outlined in this paper forms part of a study focusing on the issues of applying system identification to unmanned aerial vehicles (UAVs) ${ }^{(11)}$. Some UAVs have more unusual configurations in comparison to manned aircraft. One example is the typical configuration for unmanned combat aerial vehicles (UCAVs), which are designed to have a low radar signature and consequently have a blended wing-body and no fin. The lack of a fin and, thus, a conventional rudder, means that the aircraft requires an alternative means of yaw control $^{(12)}$. One solution is to use aerodynamic devices which generate differential drag between the wings and produce the required yawing motion. However, these devices will also change the lift generated by each wing resulting in undesirable rolling moments and, depending on the location of the devices relative to the aircraft's centre of gravity, pitching moments. Application of model structure determination to flight test data could help quantify the significance of such effects.

In this paper, two model structure determination techniques are examined. The first is stepwise regression and the second is an adaptation of Milne and Tischler's technique. Like Milne and Tischler's method, the modified approach presented in this paper utilises the estimated standard deviations, parameter insensitivity and correlation among the parameters to assess the adequacy of the model structure but parameter estimation is performed using maximum likelihood estimation, rather than the frequency response method. The two techniques are then applied to data from two sources. The first is a set of UAV flight test data, supplied by BAE Systems, and the second is data taken from dynamic wind tunnel tests on a UAV configuration. By comparing the resulting model structures from the maximum likelihood approach with that obtained from the more-established stepwise regression technique, an assessment can be made on the performance of the maximum likelihood method. While the model structure determination process has been highlighted above as being particularly useful for the analysis of nonlinear dynamics or unconventional configurations, it should be noted that the examples given in this paper involve relatively linear dynamics and relatively conventional airframe configurations. These examples have been used to validate the maximum likelihood approach for simpler, linear problems, before assessing the technique on more-complex, nonlinear data, which is the focus of further research.

The next section of the paper briefly describes the theory underlying the stepwise regression procedure, while the maximum likelihood approach is outlined in Section 3. The application of the two methods to the UAV flight test data is then presented in Section 4 , while the results of the analysis of the dynamic wind tunnel data are given in Section 5. Finally, the conclusions of the analysis are outlined in Section 6.

\subsection{STEPWISE REGRESSION}

The first model structure determination method examined in this paper is stepwise regression, which utilises least squares estimation and seeks to find the most appropriate model with the following general form, 
$z(i)=\theta_{0}+\theta_{1} X_{1}(i)+\theta_{2} X_{2}(i)+\ldots+\theta_{n} X_{n}(i)+\varepsilon(i) \quad i=1,2, \ldots, N \ldots$

where $z$ is the dependent variable, $X_{1}, X_{2}(i), \ldots, X_{n}$ are the $n$ independent variables or regressors, $\theta_{0}, \theta_{1}, \theta_{2}, \ldots \theta_{n}$ are the $m=n+1$ unknown parameters to be determined and $N$ is the number of discrete measurement points. The variable $\varepsilon$ is the so-called equation error and is assumed to account for random noise corrupting the measurement of the dependent variable. The equation error is treated as a white noise process with zero mean and variance $\sigma^{2}$. The regressors are assumed to be known without error.

If the number of available data points $N$ is greater than the number of unknown parameters to be determined $m$, the least squares estimates of the parameters are found from,

$\hat{\theta}=\left(\mathbf{X}^{\mathrm{T}} \mathbf{X}\right)^{(-1)} \mathbf{X}^{\mathrm{T}} \mathbf{z}$

where $\hat{\theta}$ is the $m \times 1$ vector containing the parameter estimates, $\mathbf{X}$ is the $\mathrm{N} \times \mathrm{m}$ matrix of data for the regressors, and $\mathbf{z}$ is the $\mathrm{N} \times 1$ vector of data for the dependent variable.

The stepwise regression process is described briefly below. More detailed theoretical treatments of stepwise regression, including equations for the various statistical metrics utilised in the method, can be found in Refs 1 and 13. The process begins with just the offset parameter $\theta_{0}$ in the aerodynamic model. The correlation between each of the regressors and $\mathbf{z}$, denoted $r_{j z}$ for the $j$ th regressor, is then calculated and the first candidate term added to the model is that with the highest value of $r_{j z}$. If the first regressor added to the model is $\mathbf{X}_{1}$ then the model structure becomes,

$\mathbf{z}=\hat{\theta}_{0}+\hat{\theta}_{1} \mathbf{X}_{1}+\mathbf{v}$

where the elements of $v$ are known as the residuals and least squares regression (Equation (2)) is used to find the parameter estimates $\theta_{0}$ and $\hat{\theta}_{1}$. The regressors subsequently added to the model are the terms which have the highest correlation with the, as yet, unmodelled dynamics i.e. the residuals. For example, the second term added to the model is the regressor which has the highest correlation with $\mathbf{z}-$ $\theta_{0}-\theta_{1} \mathbf{X}_{1}$.

Every time a regressor is added to the model, the significance of each regressor within the model is reassessed using the partial $F$ statistic, $F_{p}$. The $F_{p}$ value for each parameter is compared to a preselected $F$-distribution threshold, denoted $F_{i n}$. Any term with a value of $F_{p}$ less than $F_{i n}$ is removed from the model. It is entirely possible that a term added at an early stage of the analysis could become insignificant as more regressors are added and therefore removed at a later stage. A suitable value for $F_{\text {in }}$ is dependent on the number of data points $N$ and the number of unknown parameters $m$. From $F$ distribution look up tables (see Ref. 14, for example) a typical value for aircraft system identification problems may be $F_{\text {in }} \approx 4$. However, it has been suggested that more suitable values of $F_{\text {in }}$ are around four to five times the figure indicated by $F$-distribution tables ${ }^{(8)}$.

The sole use of the partial $F$-statistics to determine the most appropriate model structure has been found to be too restrictive and can result in the model having too many parameters. Therefore, it is recommended that a number of metrics be used in assessing the suitability of the model ${ }^{(1,8)}$. Various metrics have been proposed and the most common are summarised below.

- The coefficient of determination, denoted $R^{2}$, represents the proportion of the variation in the dependent variable explained by the terms in the model. After all significant terms have been included in the model, the change in $R^{2}$ due to the inclusion of additional terms will be small. Therefore, the appropriate model structure is considered to have been found when the increase in $R^{2}$ falls below a given threshold, typically $0.5 \%$.

- The predicted sum of squares (PRESS) is the sum of the square of the residuals in $v$, hence, in theory, the most appropriate model structure is the one with the minimum value of PRESS.
However, in practice, it has been found that for cases where the number of data points $N$ is much larger in comparison to the number of model terms $\mathrm{m}$ (which is typical for aircraft applications) then PRESS can continue to decrease even when insignificant regressors are added to the model. Therefore, care must be taken when using PRESS to assess the adequacy of the model structure.

- The predicted square error (PSE) metric is the sum of two terms, the first of which decreases as the fit to the measured data improves while the second increases as more terms are added to the model. It therefore helps in obtaining an accurate match to the data without including an excessive number of parameters in the model structure. The correct model structure is that with the minimum PSE value.

- Examination of the residuals can also provide an indication of the adequacy of the model structure. If the model structure is correct then the residuals should be a sequence of uncorrelated random variables with a Gaussian distribution. On the other hand, if they are found to contain a deterministic component, this could suggest that an important regressor has been omitted from the model.

In many cases, it is unlikely that all of the criteria described above will be satisfied at any one time. Often, the choice of when to halt the process of adding and removing terms from the model is made based on a consensus of the metrics. Another useful criterion when making a borderline decision as to whether a regressor is included or not is the principle of parsimony ${ }^{(1,2)}$, which says that given two models which have similar levels of fidelity, the better model is that which has the fewer number of parameters.

\subsection{MAXIMUM LIKELIHOOD MODEL STRUCTURE DETERMINATION}

The second model structure determination technique to be outlined in this paper is an adaptation of the approach developed by Milne and Tischler ${ }^{(3,10)}$ which, as mentioned earlier, utilises the frequency response method for identification. However, the metrics used to assess the adequacy of the model structure can be defined for other estimation techniques. In this paper, the model structure determination process is based on maximum likelihood estimation. The principal advantage time domain maximum likelihood estimation has over the frequency response identification is that system nonlinearities can be estimated directly ${ }^{(15)}$. The frequency response method, on the other hand, finds the best linear describing function to model the system. The least squares regression method described in Section 2 is also not suited for estimation when the system model is nonlinear in terms of the parameters to be estimated.

As with stepwise regression in the previous section, only a brief outline of maximum likelihood estimation will be given here. The interested reader can find detailed theoretical treatments of maximum likelihood estimation, for example, in Refs 1 and 2.

Estimates of the unknown parameters are those which minimise the cost function,

$J=\frac{1}{2} \sum_{i=1}^{N}[\mathbf{z}(i)-\mathbf{y}(i)]^{T} \mathbf{R}^{-1}[\mathbf{z}(i)-\mathbf{y}(i)]+\frac{N}{2} \ln |\mathbf{R}|$

where $\mathbf{z}$ is now a vector of measured outputs from the aircraft, $\mathbf{y}$ is a vector of predicted outputs and $\mathbf{R}$ is the covariance matrix for the noise corrupting the measurements $\mathbf{z}$. The process of determining the parameter estimates $\theta$ which minimise $J$ is an iterative one, requiring a numerical optimisation technique such as the modified NewtonRaphson method ${ }^{(16)}$.

The accuracy of the parameter estimates is defined by the parameter covariance matrix $\mathbf{P}$, which can be found approximately 
from the expression ${ }^{(2)}$,

$\mathbf{P} \approx \mathbf{M}^{-1} \approx\left\{\sum_{i=1}^{N}\left[\frac{\partial y(i)}{\partial \theta}\right]^{T} \mathbf{R}^{-1}\left[\frac{\partial y(i)}{\partial \theta}\right]\right\}^{-1}$

where $\mathbf{M}$ denotes the Fisher information matrix.

Four metrics calculated from Equation (5) are used to assess the chosen model structure:

- Standard error of the parameter estimate, denoted $s\left(\hat{\theta}_{j}\right)$ for the $j$ th parameter, and calculated from the square root of the diagonal elements of $\mathbf{P}$. The standard error approximates the standard deviation of the parameter estimate.

- Parameter insensitivity is a measure of how much a parameter value can be changed without causing an increase in the cost function. It therefore provides a measure of the significance of each parameter. For the $j$ th parameter, the insensitivity is given by,

$\mathbf{I}_{j}=1 / \sqrt{M_{j j}}$

where $\mathrm{M}_{i j}$ is the $j$ th diagonal element of the information matrix.

- The pair-wise correlation coefficient between two parameters is found from,

$$
\rho_{i j}=\frac{\mathbf{P}_{i j}}{\sqrt{\mathbf{P}_{i i} \mathbf{P}_{j j}}} i j=1,2, \ldots, m
$$

A correlation coefficient of 0 indicates that the parameters are completely independent of each other. On the other hand, a value of 1 means that the two parameters are linearly dependent and are in some way modelling the same phenomena in the aerodynamic model.

- The uncertainty or confidence ellipsoid can be used to detect correlation between more than two parameters ${ }^{(3,17)}$, which would not be shown by the pair-wise correlation coefficients from Equation (7). For the $j$ th parameter, the elements of the confidence ellipsoid are found from Ref. 3,

$$
\bar{\Theta}=\frac{\mathbf{T}^{T} \mathbf{M}_{j}^{-1}}{s\left(\widehat{\theta_{j}}\right)}
$$

where $\mathbf{T}$ is a diagonal matrix containing the insensitivities from Equation (6) and $\mathbf{M}_{j}^{(-1)}$ denotes the $j$ th column of the inverse of the information matrix. For each of the parameters, $\bar{\Theta}$ is a vector of length equal to the number of parameters, the elements of which are usually scaled to unity. For the $j$ th parameter estimate, the $j$ th element of $\bar{\Theta}_{j}$ is equal to 1 and correlation is indicated if any of the remaining elements are large in comparison to the $j$ th element.

The model structure determination process based on the above metrics is described as follows. Parameter estimation is initially carried out with all candidate terms in the model. The resulting insensitivity values are checked against a threshold and the parameter with the highest insensitivity exceeding the limit is removed from the model i.e. it is held fixed at zero or another appropriate value. Parameter estimation is then repeated. At any stage, it may be that all parameters have acceptable insensitivities but the standard errors of some parameters may be large. This is most likely due to correlation between parameters, so the correlation coefficients or confidence ellipsoid for the parameter with the largest standard error are analysed. If correlation between parameters is indicated then the choice of which parameter to remove must be made. This decision can be a difficult one and it may be useful to rely on an understanding of the physics of the aircraft to choose which term to remove. The model structure determination process is halted when all parameters have standard errors and insensitivities within the respective thresholds.
The following guidelines have been suggested for indicating if a parameter is significant enough to be included in the $\operatorname{model}^{(3)}$,

$\bar{s}_{j}=\frac{s\left(\hat{\theta}_{j}\right)}{\left|\hat{\theta}_{j}\right|} \times 100 \leq 20 \%, \bar{I}_{j}=\frac{I_{j}}{\left|\hat{\theta}_{j}\right|} \times 100 \leq 10 \%, \rho_{i j}<0 \cdot 9, \bar{\Theta}_{j}<0 \cdot 6$

It has been found from experience that the standard deviation of parameters determined from repeated manoeuvres can be greater than the theoretical values obtained from Equation (5). A discussion of this issue can be found in Refs 17 and 18. The estimated standard deviation and insensitivity thresholds suggested in Ref. 3 include a 'fudge factor' of 2 to compensate for this discrepancy. Some analysts, instead of a 'fudge factor', prefer to modify the calculation of the covariance matrix once the parameters have been estimated and, for the analysis presented in this paper, a correction proposed by Morelli and Klein ${ }^{(18)}$ has been utilised.

It should also be noted that the guidelines in Equation (9) are suggested for frequency response analysis and, because of differences in the formulation of the cost function for the two methods, may not be reliable thresholds for the maximum likelihood approach. In the examples presented in Sections 4 and 5, the thresholds in Equation (9) were used. By then comparing the resulting model structure with that obtained from the more-established stepwise regression technique, a judgement on the suitability of the thresholds for the maximum likelihood approach could be made.

In comparing the maximum likelihood method to stepwise regression, the merits and drawbacks of each technique will in part be related to the characteristics of the underlying estimation method used. Full descriptions of the advantages and disadvantages of least squares and maximum likelihood estimation can be found in Refs 1 and 2 . Least squares regression, for example, is a simple estimation method which can be solved using matrix algebra. Maximum likelihood estimation, in its most common output error formulation, on the other hand is a more complex iterative process requiring a suitable numerical optimisation algorithm to find the unknown parameters. However, least squares estimation assumes that the regressors are known without error. In practice, measurements from the aircraft will always contain a given amount of noise which will adversely affect the parameter estimates. The maximum likelihood output error method, in contrast, directly accounts for noise in the recorded motion variables. In terms of the model structure determination process itself, one advantage that stepwise regression has over the maximum likelihood approach described above is that, at each stage of the process, the significance of terms already included in the model are reassessed. Therefore, as already described, if a term added early on in the process is found be less significant as more parameters are added to the model, it can subsequently be removed. The maximum likelihood approach conversely can be thought of as a 'backward elimination' process. All terms are initially included in the model and insignificant terms are removed one at a time until an appropriate model structure has been obtained. However, there is no mechanism in the process to re-introduce parameters which may have been considered insignificant early in the process but whose importance only becomes evident as further terms are removed from the model structure. This could potentially be an issue if using the maximum likelihood approach for complex nonlinear problems with large numbers of candidate parameters.

\subsection{UAV FLIGHT TEST DATA EXAMPLE}

The results presented in this section are for the analysis of UAV flight test data in order to determine an aerodynamic model for the aircraft's rolling moment. The analysis was carried out on six concatenated data segments containing a series of aileron and rudder inputs, as shown in Fig. 1. 

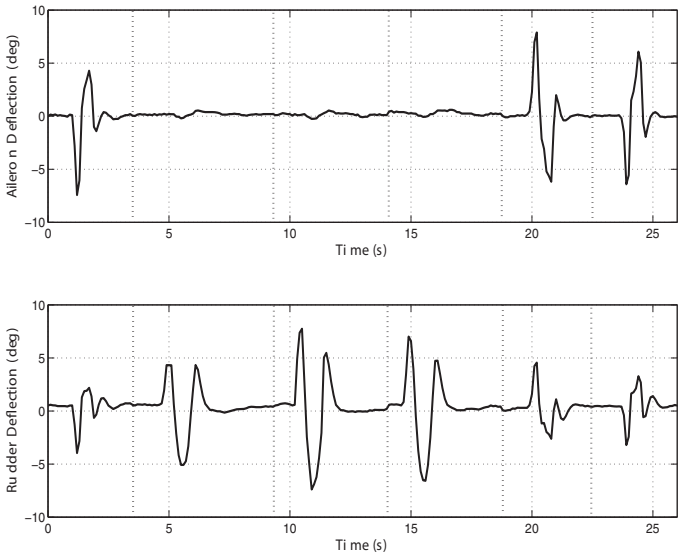

Figure 1. Time histories of aileron and rudder deflections.

The dependent variable is the non-dimensional rolling moment coefficient $\mathrm{Cl}$ defined as,

$$
C l=\frac{L}{\frac{1}{2} \rho V^{2} S s}
$$

where $L$ is the dimensional rolling moment, $\rho$ is the atmospheric density, $V$ is the aircraft's true airspeed, $S$ is its wing area and $s$ its wing semi-span. This non-dimensional notation system was chosen for the analysis because it matched the notation system used in an existing simulation model of the aircraft and, ultimately, one of the purposes of the system identification analysis was to validate the derivative values within this simulation model.

As mentioned in the Introduction, the UAV from which the data was obtained can be considered to be relatively conventional in configuration. From empirical analysis methods, such as those published by the Engineering Science Data Unit ${ }^{(19)}$ (ESDU) or DATCOM $^{(20)}$, the following regressors were considered as the most likely to be significant for the manoeuvres under analysis,

$\beta \quad$ angle-of-sideslip

$\hat{p} \quad$ non-dimensional roll rate $(p s / \mathrm{V})$

$\hat{r}$ non-dimensional yaw rate $(r s / \mathrm{V})$

$\xi \quad$ aileron deflection

$\zeta \quad$ rudder deflection

where $p$ and $r$ are the dimensionalised roll and yaw rates respectively.

Note that, for commercial reasons, the aerodynamic derivative values from the UAV flight test data cannot be presented. Instead, the parameter estimation values are expressed as ratios of the flight test estimates to the empirically-derived estimates. For example, the lateral stability derivative estimates are expressed in the results sections below as,
$\overline{C_{l \beta}}=\frac{C_{l \beta}^{F}}{C_{l \beta}^{E}}$

where superscripts $F$ and $E$ denote flight test estimate and empirical derivative values respectively.

\subsection{Stepwise regression analysis}

For the first step of the analysis, the correlation $r_{j z}$ between each of the regressors and the rolling moment coefficient $C_{1}$ were calculated and are shown in the first section of Table 1 . The offset term $\mathrm{Cl}_{0}$ was included in the model by default. Also displayed are the initial values for the fit error $\hat{\sigma}^{2}$, the coefficient of determination $R^{2}$, PRESS and PSE. The regressor with the largest correlation with the dependent variable was the aileron deflection $\xi$, therefore the control derivative $\mathrm{Cl}_{\xi}$ was the first term added to the model.

In Step 2 of the analysis, least squares estimation was used to find the value of $\mathrm{Cl}_{\xi}$. This is given in the second section of Table 1 . The partial $F$-statistic for the derivative was calculated to be $160 \cdot 94$. For the analysis presented here, parameters were considered to be significant if their partial $F$-statistic exceeded a value of $F_{i n}=20$. With the inclusion of $\mathrm{Cl}_{\xi}$ in the model, the values of $\hat{\sigma}^{2}$, PRESS and PSE all decreased in comparison to step 1 and $R^{2}$ increased to $38.23 \%$. The values of $r_{j z}$ for the remaining candidate regressors were calculated and the next most significant term was found to be $\mathrm{Cl}_{p}$.

The third section of Table 1 shows the results of adding the roll damping derivative to the model. Its $F_{p}$ value was found to be 310.90, while the same statistic for $\mathrm{Cl}_{\xi}$ increased to 663.64. Again, the inclusion of $\mathrm{Cl}_{p}$ led to decreases in the fit error, PRESS and PSE, while the coefficient of determination rose to $71.93 \%$. Of the remaining terms, the yaw rate $\hat{r}$ was found to have the highest correlation to the as yet unmodelled effects in $\mathrm{Cl}$, therefore $\mathrm{Cl}_{r}$ was the next derivative added to the model.

The results of Step 4 of the analysis are displayed in Table 2. The new derivative $\mathrm{Cl}_{r}$ had a partial $F$-statistic of 74.75 and its addition to the model resulted in $R^{2}$ increasing to $78 \cdot 24 \%$. The other metrics $\hat{\sigma}^{2}$, PRESS and PSE also continued to decrease. The correlation values with the dependent variable for the two remaining candidate regressors, $\beta$ and $\zeta$, suggested that sideslip angle was the more significant term, hence it was included in the model next.

The addition of $\mathrm{Cl}_{\beta}$ to the model resulted in further reductions in the fit error, PRESS and PSE metrics and the coefficient of determination increased to $83.58 \%$, as shown in Table 2 . The value of $F_{p}$ for $\mathrm{Cl}_{\beta}$ was calculated to be 83.73 and the partial $F$-statistics for the other model parameters also increased with the addition of $\mathrm{Cl}_{\beta}$.

In the sixth step of the process, the final candidate regressor $\zeta$ was added to the model and the results are displayed in the final section of Table 2. This time, the partial $F$-statistic of the new parameter $\mathrm{Cl}_{\zeta}$ was found to be 5.41, which was below the $F_{\text {in }}$ value of 20 . As well as this, the PSE value increased slightly from $2.371 \times 10^{-6}$ to

Table 1

Stepwise regression steps 1 to 3

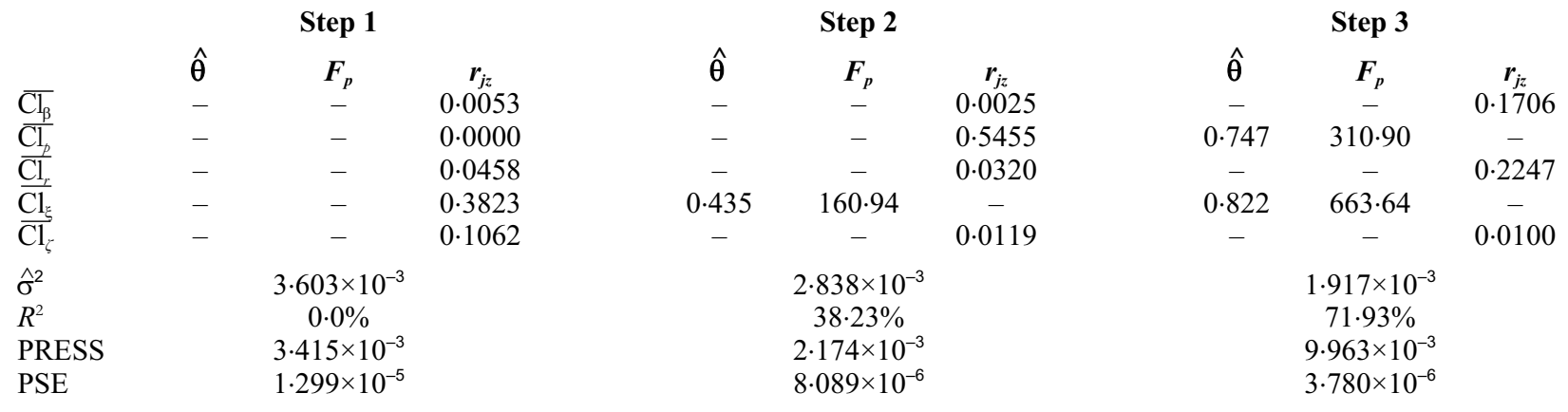


Table 2

Stepwise regression steps 4 to 6

\begin{tabular}{|c|c|c|c|c|c|c|c|c|}
\hline & & Step 1 & & & Step 2 & & & Step 3 \\
\hline & $\hat{\theta}$ & $F_{p}$ & $r_{j z}$ & $\hat{\theta}$ & $F_{p}$ & $r_{j z}$ & $\hat{\theta}$ & $\boldsymbol{F}_{p}$ \\
\hline$\overline{\mathrm{Cl}_{\beta}}$ & - & - & $0 \cdot 2457$ & $1 \cdot 320$ & $83 \cdot 73$ & - & $1 \cdot 251$ & $73 \cdot 11$ \\
\hline$\overline{\mathrm{Cl}_{p}}$ & $0 \cdot 807$ & $450 \cdot 76$ & - & 0.944 & $676 \cdot 07$ & - & 0.959 & $688 \cdot 15$ \\
\hline$\overline{\mathrm{Cl}}$ & 1.934 & 74.75 & - & $2 \cdot 020$ & $107 \cdot 50$ & - & $2 \cdot 108$ & 114.68 \\
\hline $\overrightarrow{\mathrm{Cl}_{\xi}}-$ & 0.831 & 871.04 & - & 0.911 & $1226 \cdot 6$ & - & 0.936 & $1,119 \cdot 4$ \\
\hline$\overline{\mathrm{Cl}_{\zeta}}$ & - & - & 0.0504 & - & - & $0 \cdot 0207$ & $0 \cdot 390$ & $5 \cdot 41$ \\
\hline$\hat{\sigma}^{2}$ & & $1.691 \times 1^{-}$ & & & $1.471 \times 10$ & & & $1.459 \times 10^{-3}$ \\
\hline$R^{2}$ & & $78.24 \%$ & & & $83.58 \%$ & & & $83.92 \%$ \\
\hline PRESS & & $7.775 \times 10$ & & & $5.921 \times 10^{-}$ & & & $5.827 \times 10^{-3}$ \\
\hline PSE & & $3.014 \times 10$ & & & $2 \cdot 371 \times 10$ & & & $2 \cdot 377 \times 10^{-6}$ \\
\hline
\end{tabular}

Table 3

Results for maximum likelihood analysis of the rolling moment equation

\begin{tabular}{|c|c|c|c|c|c|c|c|c|c|}
\hline & \multicolumn{3}{|c|}{ Step 1} & \multicolumn{3}{|c|}{ Step 2} & \multicolumn{3}{|c|}{ Step 3} \\
\hline & $\hat{\theta}$ & $\bar{s}$ & $\bar{I}$ & $\hat{\theta}$ & $\bar{s}$ & $\bar{I}$ & $\hat{\theta}$ & $\bar{s}$ & $\bar{I}$ \\
\hline$\overline{\mathrm{Cl}_{\beta}}$ & $1 \cdot 644$ & $12 \cdot 40$ & $6 \cdot 02$ & 1.687 & $7 \cdot 52$ & $5 \cdot 87$ & 1.747 & 8.58 & $5 \cdot 67$ \\
\hline$\overline{\mathrm{Cl}} \mathrm{p}_{p}$ & 1.220 & $6 \cdot 05$ & 1.70 & $1 \cdot 201$ & $5 \cdot 88$ & 1.72 & 1.201 & $5 \cdot 88$ & 1.72 \\
\hline $\overrightarrow{\mathrm{Cl}_{r}}$ & 1.969 & 8.41 & 6.69 & 1.962 & $8 \cdot 52$ & $6 \cdot 72$ & 1.874 & $9 \cdot 61$ & $7 \cdot 04$ \\
\hline$\overline{\mathrm{Cl}_{\xi}}$ & 1.061 & 4.65 & $1 \cdot 33$ & 1.060 & 4.68 & $1 \cdot 34$ & 1.028 & $4 \cdot 50$ & $1 \cdot 38$ \\
\hline$\overline{\mathrm{Cl}_{\zeta}}$ & 0.564 & $36 \cdot 00$ & 21.94 & $0 \cdot 547$ & $35 \cdot 28$ & 22.69 & $0^{*}$ & - & - \\
\hline$\overline{\mathrm{Cl}}_{t 0}$ & $1 \cdot 210$ & $212 \cdot 71$ & $140 \cdot 21$ & $0^{*}$ & - & - & $0^{*}$ & - & - \\
\hline
\end{tabular}

* - Removed from model structure

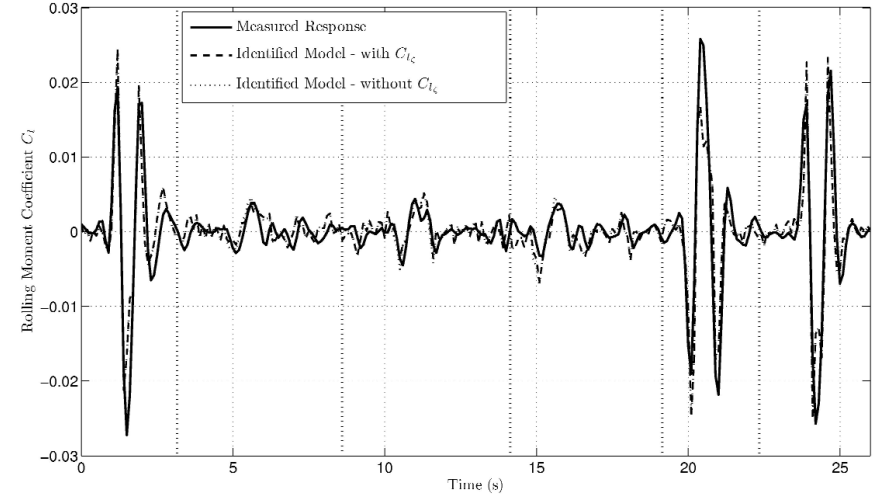

Figure 2. Comparison of measured and identified rolling moment time histories - with and without $\mathrm{Cl}_{\zeta}$.

$2 \cdot 377 \times 10^{-6}$. The addition of $\mathrm{Cl}_{\zeta}$ to the model resulted in $R^{2}$ increasing to $83.92 \%$, however this rise in comparison to the value in the previous step was only $0.34 \%$, which fell below the guideline of a minimum increase of $0.5 \%$ in the coefficient of determination for a new regressor to be considered significant. On the other hand, the estimated fit error $\hat{\sigma}^{2}$ decreased, as did the PRESS metric. However, as explained above, for typical aircraft problems the value of PRESS can continue to decrease even when an insignificant regressor is added to the model. Time histories of the rolling moment coefficient derived from aircraft measured data and the response generated using the identified model, with and without the inclusion of $\mathrm{Cl}_{\xi}$, are shown in Fig. 2. It can be seen that there is no significant change in the identified response when $\mathrm{Cl}_{\zeta}$ is added or removed from the model. Therefore, taking all the metrics into account and considering the principle of parsimony introduced earlier, it was decided that the derivative $\mathrm{Cl}_{\zeta}$ should not be included in the model. The final rolling moment equation was then,
$\mathrm{Cl}=\mathrm{Cl}_{\beta} \beta+\mathrm{Cl}_{p} \hat{p}+\mathrm{Cl}, \hat{r}+\mathrm{Cl}_{\xi} \xi$

Note that the offset term $\mathrm{Cl}_{\circ}$ was itself found to be negligibly small so was removed from the model.

\subsection{Maximum likelihood analysis}

The analysis begins with all candidate terms in the model so the initial equation for the rolling moment was taken to be,

$\mathrm{Cl}=\mathrm{Cl}_{0}+\mathrm{Cl}_{\beta} \beta+\mathrm{Cl}_{p} \hat{p}+\mathrm{Cl}, \hat{r}+\mathrm{Cl}_{\xi} \xi+\mathrm{Cl}_{\zeta} \zeta$

The results for Step 1 are given in the first section of Table 3, which shows the estimated derivative values, the standard errors and the insensitivities. Of the six parameters, the offset term $\mathrm{Cl}_{0}$ had by far the largest insensitivity at $140 \cdot 21 \%$ so was removed from the model structure. Parameter estimation was repeated and the results are displayed in the middle section of Table 3 . This time, $\mathrm{Cl}_{\xi}$ was found to have the highest insensitivity at $22.69 \%$ so this parameter was also removed from the model. On the third run, the standard errors were all found to be below $20 \%$ and all insensitivities were under $10 \%$. Therefore the procedure was halted at this point, with the final model structure given by,

$\mathrm{Cl}=\mathrm{Cl}_{\beta} \beta+\mathrm{Cl}_{p} \hat{p}+\mathrm{Cl}_{,} \hat{r}+\mathrm{Cl}_{\xi} \xi$

This matched the model structure determined using stepwise regression, given in Equation (12), indicating that the respective thresholds of $20 \%$ and $10 \%$ for the standard errors and insensitivities were reliable guidelines for this particular example. Comparison of the final parameter estimates in Table 3 with those in Table 2 showed that the maximum likelihood values for $\mathrm{Cl}_{r}$ and $\mathrm{Cl}_{\xi}$ matched the stepwise regression values to within around $10 \%$. The discrepancies in the $\mathrm{Cl}_{\beta}$ and $\mathrm{Cl}_{p}$ estimates were slightly larger at around $30 \%$.

Figure 3 shows a comparison of the time histories of $\mathrm{Cl}$ identified using the two analysis techniques. While the general match between 


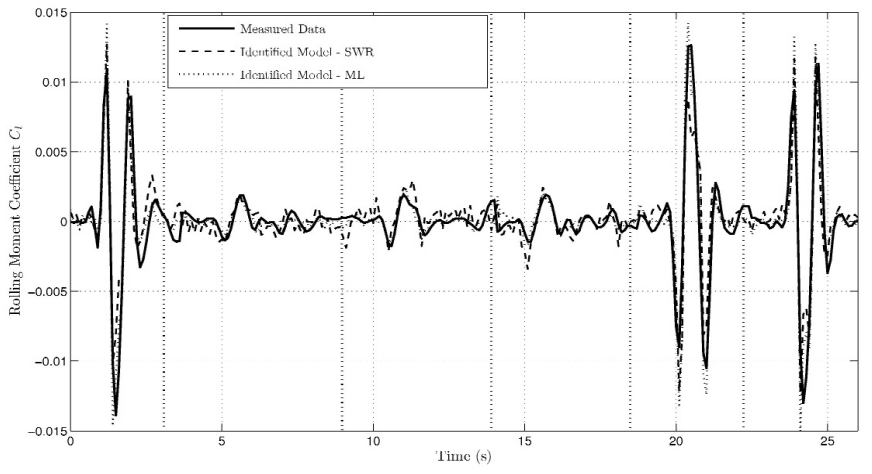

Figure 3. Comparison of identified rolling moment time histories from stepwise regression and maximum likelihood analysis.
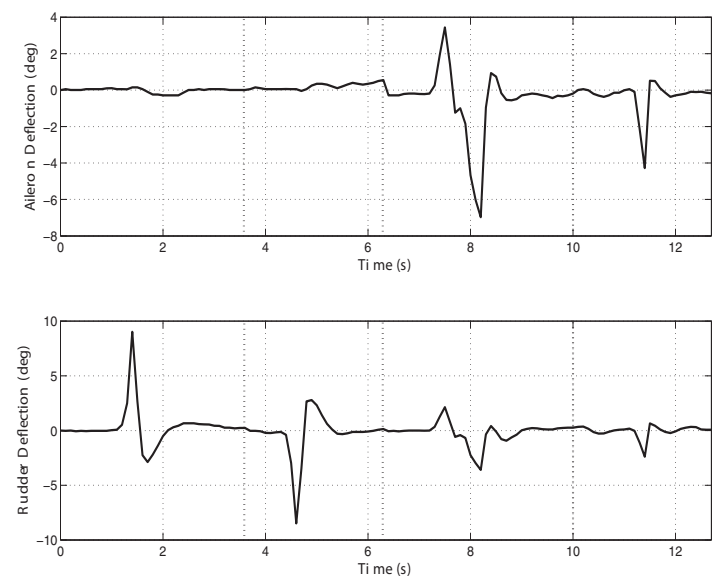

Figure 4. Time histories of aileron and rudder deflections for validation manoeuvres.

the measured and identified responses may be considered close, discrepancies can be seen around some of the peaks during the aileron inputs. The stepwise regression model tends to under-predict the rolling moment response while the maximum likelihood model over-predicts the rolling moment in these regions. This may be due to differences in the estimated values of the derivative $\mathrm{Cl}_{p}$. Significant roll rates would result from the aileron inputs and, as mentioned above, the maximum likelihood estimate of $\mathrm{Cl}_{p}$ is around $30 \%$ greater than the stepwise regression estimate. The difference between the two estimates of $\mathrm{Cl}_{\xi}$ is much smaller in comparison. As to why the identified models do not match the measured data in these regions, the parameter estimation process is trying to find a single value for each derivative which provides the best match over the six manoeuvres. In practice, the derivative values will vary with flight conditions. However, there were no significant changes in variables such as airspeed or angle of attack over the six manoeuvres which might have explained why the identified models match the data more closely in some regions and not so well in others. Further investigation of the data is required to identify the cause of these discrepancies.

The identified models were also subsequently used to predict the aircraft response using a set of independent data not used in the estimation process. The data used to validate the models comprised four concatenated segments containing aileron and rudder inputs, as shown in Fig. 4. The rolling moment response predicted by the models are plotted against the measured data in Fig. 5. The match provided by the models to the data is of a similar fidelity to that in Fig. 3.

A criticism of the validation process presented here could be that the inputs used for validation were similar in nature to those used for the identification process (Fig. 1). Ideally, the validation inputs should be dissimilar, or have a different frequency content, from the

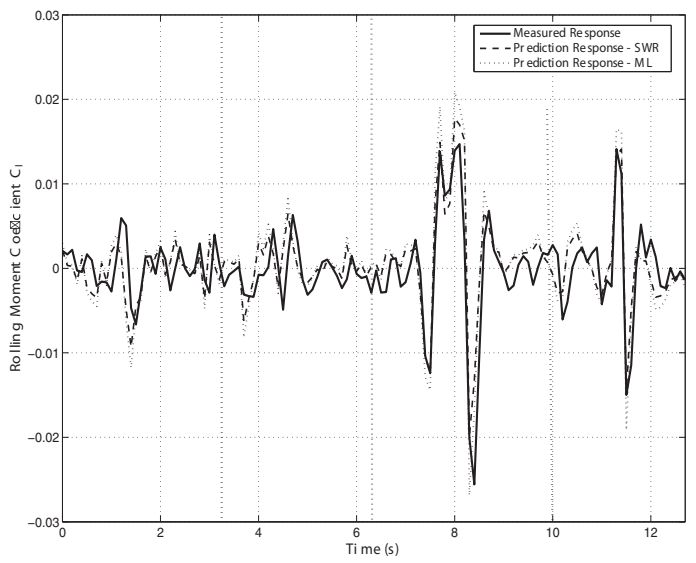

Figure 5. Measured and predicted rolling moment time histories for validation manoeuvres.

identification inputs to ensure against the identified model providing adequate predictive capability for only one particular input type ${ }^{(3)}$. For example, frequency sweeps could be used for identification and doublets could be used for validation. Unfortunately, inputs of dissimilar nature were not available for this analysis.

As pointed out at the start of this section, empirical analysis of this UAV indicated that the rudder contribution to rolling moment was significant. However, both stepwise regression and maximum likelihood analysis indicated $\mathrm{Cl}_{\zeta}$ to be small and that the parameter should be excluded from the model. As mentioned in the Introduction, part of the system identification process is the design of control surface inputs to adequately excite the aircraft dynamics of interest and the model structure determination process will only highlight as significant the aerodynamic derivatives which can be reliably identified from the data. The subject of optimal design of control surface inputs is discussed in Refs 1 and 2. It could be that the data analysed simply did not contain enough information about $\mathrm{Cl}_{\zeta}$. However, significant rudder inputs were applied during the manoeuvres flown in flight, as shown in Figs 1 and 4, and in the regions of rolling moment response corresponding to these rudder inputs, there was little change in the value of $\mathrm{Cl}$, as shown in Figs 3 and 5. Therefore, based on the available flight test data, it is concluded that the rolling moment due to rudder is not significant for this aircraft.

\subsection{WIND-TUNNEL TEST DATA EXAMPLE}

The wind tunnel data used in this paper were obtained from tests performed at Cranfield University's dynamic wind tunnel facility, which allows scaled aircraft models with actuated control surfaces to be flown in semi-free flight, emulating flight test-like experiments. The commands to the control surfaces and the aircraft model's resulting motion are measured, allowing system identification to be performed. A miniature inertial measurement unit is housed within the model to record its motion. A detailed description of the facility can be found in Refs 11 and 21 .

The data analysed in this example comes from tests of an approximate $1 / 3$ scale model of a demonstrator UAV (shown in Fig. 6) developed for the Flapless Air Vehicle Integrated Industry Research (FLAVIIR) programme ${ }^{(22)}$, which is jointly funded by BAE Systems and the Engineering and Physical Sciences Research Council (EPSRC). The broad aim of the project, which is a collaboration between ten university partners, is to develop technologies for UAVs with unconventional control surfaces.

During the tests, the model was restricted to three degrees of freedom (DoF) - roll, pitch and yaw. The data analysed here contained a doublet input to the rudder. The time history of the 


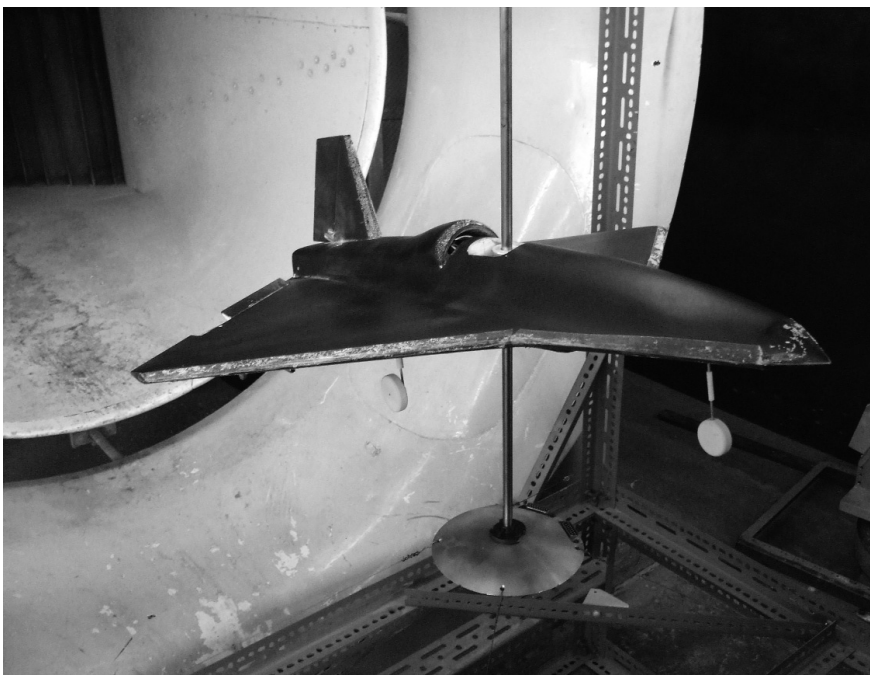

Figure 6. 1/3 Scale model of the FLAVIIR demonstrator UAV.

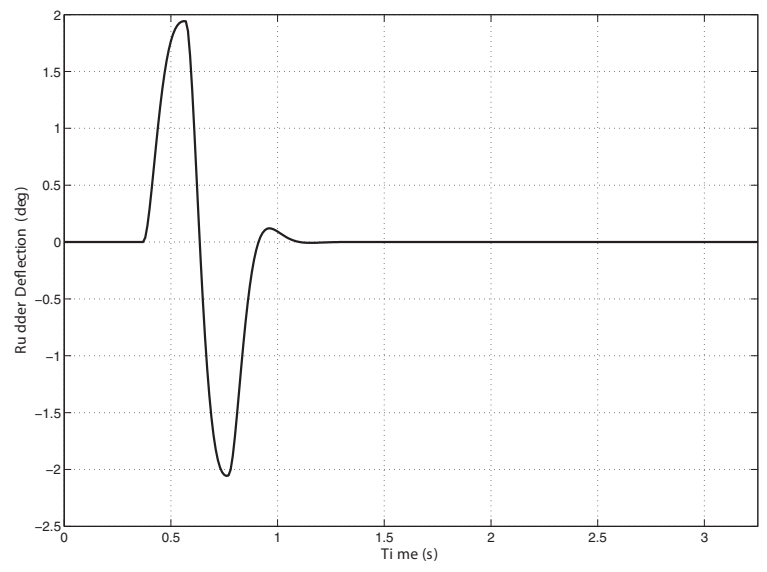

Figure 7. Rudder deflection angle for doublet input.

rudder deflection angle is shown in Fig. 7. A theoretical investigation of the motion of aircraft models on the test rig indicated that the following state space representation could be used to describe the motion as a result of a rudder input ${ }^{(10)}$,

$$
\left[\begin{array}{c}
\dot{v} \\
\dot{r}
\end{array}\right]=\left[\begin{array}{ll}
0 & y_{r} \\
n_{v} & n_{r}
\end{array}\right]\left[\frac{v}{r}\right]+\left[\frac{0}{n_{\zeta}}\right] \zeta
$$

where $v$ is the lateral velocity component, $r$ is the yaw rate and $\zeta$ is the rudder deflection. The sideforce derivative $y_{r}$ and the yawing moment derivatives $n_{v}, n_{r}$ and $n_{\zeta}$ are in the concise form defined in $\mathrm{Cook}^{(24)}$. Note that Equation (15) utilises a different notation system from that used in Section 4. The reason for this is that, as with the UAV flight test example, one of the purposes of the system identification analysis was to validate the derivative values in an existing simulation model. This simulation model utilised the state space representation given in Equation (15), therefore, the analysis was also carried out using the state space form.

Equation (15) is effectively the classical second-order dutch roll approximation. However, the rig suppresses the lateral translational motion of the aircraft model, resulting in the theoretical values of the sideforce derivatives with respect to $v$ and $\zeta$ being zero. The model will see a lateral velocity component $v$ due to yaw or sideslip. The value of the sideforce derivative $y_{r}$ should in theory have a value of approximately the negative of the wind tunnel speed $V_{T}$, which, for this example, was $30 \mathrm{~ms}^{-1}$.

During the test, the aircraft model was observed to undergo some
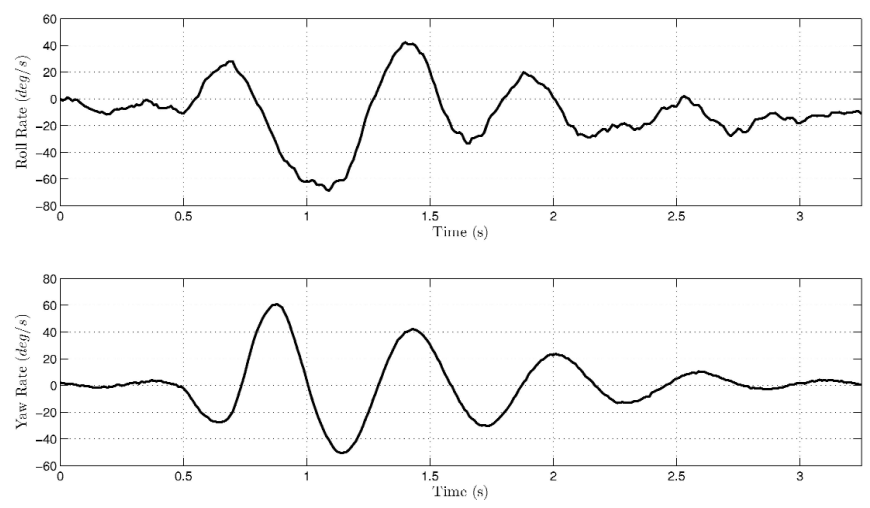

Figure 8 . Roll rate and yaw rate time histories.
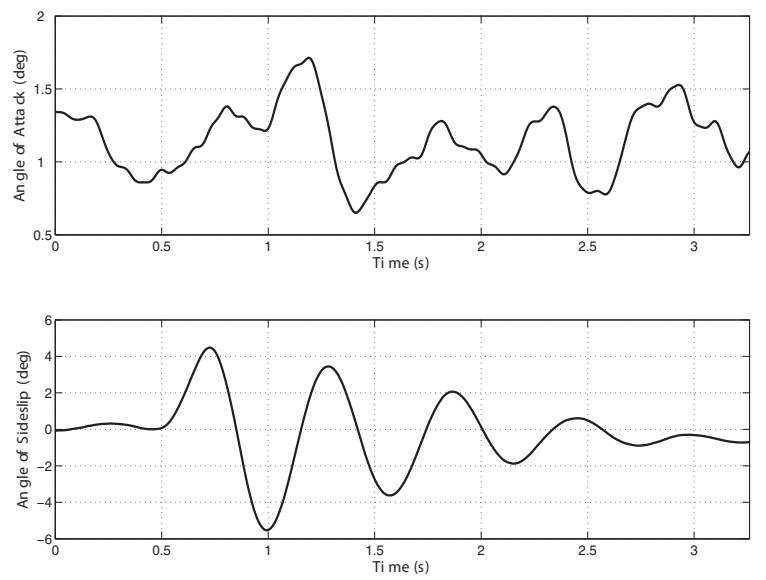

Figure 9. Angle-of-attack and angle-of-sideslip time histories.

rolling motion when the rudder input was applied. This can be seen in Fig. 8 which shows that the model experienced a roll rate oscillation of a similar magnitude to the yaw rate oscillation. The model was also free to rotate in pitch, allowing changes in angle of attack to occur during the manoeuvre. Figure 9 shows that some variation in angle of attack, between approximately 0.5 and 2 degrees, was observed. Equation (15) is based on the assumption of small perturbations about an initial trim condition and that longitudinal and lateral/directional dynamics are decoupled i.e. for $a$ lateral/directional perturbation, angle-of-attack is assumed to be constant. The candidate model structure, therefore, could have been extended in this example to account for these changes in incidence. However, the angle-of-attack was limited to relatively low angles over a narrow range. Hence, in a first instance, it was assumed that the lateral/directional dynamics would be insensitive to these changes of angle-of-attack over this range and an angle-of-attack dependency was not included in the candidate model structure.

The data acquisition process also lead to the introduction of a time delay between the rudder data and the other motion variables, denoted $\tau_{\zeta}$. For the data being analysed here, the value of $\tau_{\zeta}$ was known to be around $0.28 \mathrm{sec}$. With the inclusion of rolling motion effects and the time delay, the initial model structure containing all candidate terms was assumed to take the following form,

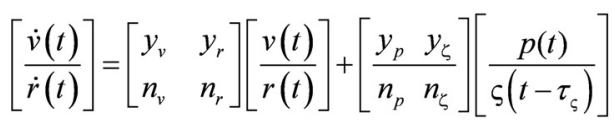

Note that the roll rate $p$ has been included in Equation (16) not as a state variable but as, what is termed, a pseudo-control input ${ }^{(2)}$. This approach allows the effects of additional motion variables to be incorporated into the equations of motion without increasing the model complexity. 
Table 4

Stepwise regression Steps 1-3 for sideforce equation

\begin{tabular}{|c|c|c|c|}
\hline & $\hat{\theta}$ & $\underset{F_{p}}{\text { Step } 1}$ & $r_{j z}$ \\
\hline$y_{v}$ & - & - & $0 \cdot 0000$ \\
\hline$y_{p}$ & - & - & $0 \cdot 0854$ \\
\hline$y_{r}$ & - & - & 0.9986 \\
\hline$y_{\zeta}$ & - & - & $0 \cdot 3272$ \\
\hline$\sigma^{2}$ & & $10 \cdot 3625$ & \\
\hline$R^{2}$ & & $0.0 \%$ & \\
\hline PRESS & & $3.758 \times 10^{4}$ & \\
\hline PSE & & $107 \cdot 38$ & \\
\hline
\end{tabular}

$\begin{array}{ccc}\hat{\boldsymbol{\theta}} & \text { Step 2 } & \\ - & \boldsymbol{F}_{\boldsymbol{p}} & \boldsymbol{r}_{\boldsymbol{j} z} \\ - & - & 0 \cdot 0000 \\ -29.05 & - & 0 \cdot 1372 \\ - & 252,500 & - \\ & - & 0 \cdot 1877 \\ & 0.3845 & \\ & 99 \cdot .86 \% & \\ & 52.343 & \\ & 0.762 & \end{array}$

$\begin{array}{ccc}\hat{\boldsymbol{\theta}} & \text { Step 3 } & \\ - & \boldsymbol{F}_{\boldsymbol{p}} & \boldsymbol{r}_{\boldsymbol{j} z} \\ - & - & 0 \cdot 1706 \\ -28 \cdot 74 & - & 0 \cdot 0399 \\ 0 \cdot 647 & 208,500 & - \\ & 79 \cdot 97 & - \\ & 0 \cdot 3470 & \\ & 99 \cdot 89 \% & \\ & 42 \cdot 810 \\ & 1.042\end{array}$

Table 5

Stepwise regression Steps 1-3 for yawing moment equation

\begin{tabular}{|c|c|c|c|}
\hline & $\hat{\theta}$ & $\underset{F_{p}}{\text { Step } 1}$ & $\boldsymbol{r}_{j z}$ \\
\hline$n_{v}$ & - & - & $0 \cdot 8477$ \\
\hline$n_{p}$ & - & - & $0 \cdot 2840$ \\
\hline$n_{r}$ & - & - & $0 \cdot 0000$ \\
\hline$n_{\zeta}$ & - & - & 0.0545 \\
\hline$\sigma^{2}$ & & $\begin{array}{c}4.0250 \\
0.0^{\circ}\end{array}$ & \\
\hline PRESS & & $5,670 \cdot 4$ & \\
\hline PSE & & $16 \cdot 201$ & \\
\hline
\end{tabular}

$\begin{array}{ccc}\hat{3} & \text { Step 3 } & \\ \hat{\boldsymbol{\theta}} & \boldsymbol{F}_{\boldsymbol{p}} & \boldsymbol{r}_{\boldsymbol{j} z} \\ 3.759 & 3,659.4 & - \\ - & - & 0.0495 \\ - & - & 0.3949 \\ -3.448 & 299.0 & - \\ & 1.5537 & \\ & 91.83 \% & \\ & 476.81 & \\ & 1.4588\end{array}$

Table 6

Stepwise regression Steps 4-5 for yawing moment equation

\begin{tabular}{|c|c|c|c|c|c|}
\hline & $\hat{\theta}$ & $\underset{F_{p}}{\text { Step }} 4$ & $\boldsymbol{r}_{j z}$ & $\hat{\theta}$ & $\begin{array}{c}\text { Step } 5 \\
F_{p}\end{array}$ \\
\hline$y_{v}$ & $3 \cdot 776$ & $6,082 \cdot 3$ & - & $3 \cdot 281$ & $1,963 \cdot 0$ \\
\hline$y_{p}$ & - & - & $0 \cdot 1682$ & 1.967 & $69 \cdot 55$ \\
\hline$y_{r}$ & -2.447 & $225 \cdot 2$ & - & $-3 \cdot 702$ & $305 \cdot 6$ \\
\hline$n_{\zeta}$ & $-5 \cdot 021$ & $717 \cdot 9$ & - & $-6 \cdot 295$ & 752.9 \\
\hline$\sigma^{2}$ & & 0.8987 & & & $0 \cdot 8209$ \\
\hline$R^{2}$ & & $95.06 \%$ & & & $95 \cdot 89 \%$ \\
\hline PRESS & & $290 \cdot 33$ & & & $240 \cdot 96$ \\
\hline PSE & & $0 \cdot 9841$ & & & $0 \cdot 8963$ \\
\hline
\end{tabular}

There are a number of ways of dealing with time delays. The simplest approach would be to manually time shift the rudder data to match the other measurements. However, time delays can also be treated as unknown parameters to be estimated along with the aerodynamic derivatives. In the stepwise regression analysis, presented next, the time delay was removed manually prior to the model structure determination process. In the maximum likelihood example in Section 5.2, the time delay was included as a parameter to be estimated. Note that the parameter estimation values presented in the following sections have not been normalised (unlike Sections 4.1 and 4.2 ) but are those obtained directly from the analysis.

\subsection{Stepwise regression analysis}

For the stepwise regression analysis, the side force and yawing moment equations are treated separately. Concentrating first on the sideforce equation, the stepwise regression results are displayed in Table 4. To start with, yaw rate $r$ was found to be the regressor most significantly correlated with $\dot{v}$, hence was the first term added to the model. When this was done, a very close match to the measured data was achieved, with the coefficient of determination rising to $99.86 \%$.
The next most significant regressor was the rudder input so $y_{\zeta}$ was also added to the model. However, the increase in $R^{2}$ as a result was only $0.03 \%$. There was also a rise in the PSE statistic. The partial $F$ statistic of $y_{\zeta}$ did exceed the $F_{i n}$ threshold of 20 (79.97 from Table 4) but was significantly smaller than the $F_{p}$ figure for $y_{r}$. Therefore, $y_{\zeta}$ was removed from the equations of motion.

Tables 5 and 6 present the results from the analysis of the yawing moment equation. The regressor with the highest correlation to yaw acceleration $\dot{r}$ was the sideslip velocity $v$, therefore $n_{v}$ was the first derivative added to the model. The next term included was $n_{\zeta}$ and this was followed by $n_{r}$, leaving roll rate $p$ as the only remaining candidate regressor. When $n_{p}$ was added to the model, the improvement in the coefficient of determination was marginal, with $R^{2}$ increasing by $0.83 \%$. However, this is greater than the $0.5 \%$ guideline suggested in Section 2. Also, the partial $F$-statistic for $n_{r}$ was greater than $F_{\text {in }}$ and its inclusion in the model structure led to decreases in PRESS and PSE. Therefore, it was a borderline decision as to whether $n_{p}$ should be included in the equations of motion. Figure 10 displays the measured time history of $\dot{r}$ plotted against those from the identified model with and without $n_{p}$ in the model structure. As can be seen, the inclusion of the derivative made little 


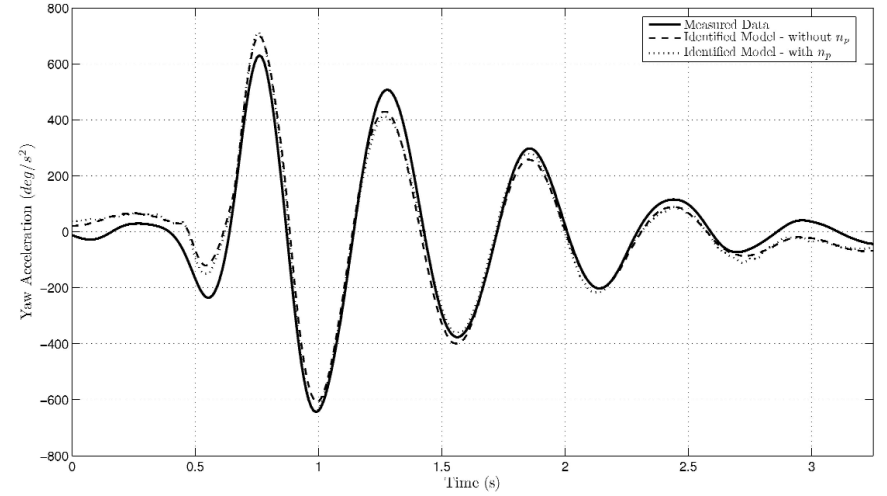

Figure 10. Comparison of identified yaw responses with and without $n_{p}$ in model structure.

difference to the reconstructed response, despite significant levels of roll rate, as shown in Fig. 8. It may be that the contribution to yawing moment due to roll rate had already been accounted for through the yaw variation with sideslip velocity, as the two effects are coupled aerodynamically ${ }^{(24)}$. A disturbance in sideslip will result in differential lift and induced drag across the wing span, leading to rolling and yawing motion. The rolling motion itself will result in the port and starboard wings seeing different levels of induced drag, also contributing to the yawing moment. Therefore, the two effects may be difficult to separate. Considering the principle of parsimony and the fact that the theoretical equations of motion due to a rudder input (Equation (15)) did not include the parameter, it was felt that the most appropriate model structure was one without $n_{p}$.

\subsection{Maximum likelihood analysis}

The choice was made to perform the maximum likelihood analysis in the frequency domain, an outline of which can be found in Ref. 1. In comparison to time domain approaches, the frequency domain is better suited to the estimation of time delays, such as $\tau_{\zeta}{ }_{\zeta}^{(3,25)}$.

The results of the analysis are displayed in Tables 7 and 8 . The control derivative $y_{\zeta}$ was found to have the highest insensitivity at $21.62 \%$ so was the first parameter removed from the model. When the estimation process was repeated, all the parameters were found to have insensitivities below the $10 \%$ guideline, as shown in second section of Table 7. However $y_{v}, y_{p}, n_{p}$ and $n_{r}$ had standard errors greater than $20 \%$. The derivative with the lowest confidence was $n_{p}$, which had a standard error of $76.88 \%$, therefore the correlation coefficients and confidence ellipsoid for this parameter were analysed. These are shown in the fourth and fifth columns respectively of the second section of Table 7 . None of the correlation coefficients exceeded 0.9 but the values of $\rho_{n p}$ were still relatively high $(\approx 0.8)$ between $n_{p}$ and the other yawing moment derivatives $n_{v}$, $n_{r}$ and $n_{\zeta}$. The elements of the confidence ellipsoid also suggested that some correlation existed between $n_{p}$ and other parameters, particularly $n_{v}$ and $n_{r}$, for which the absolute values of $\bar{\Theta}_{n p}$ were greater than $0 \cdot 6$. These correlation figures may be due to the strong aerodynamic coupling between roll rate, yaw rate and sideslip velocity on yawing moment, described earlier in Section 5.1. The choice was made to remove $n_{b}$ from the model. The confidence in the estimated value of this derivative was low, despite the magnitude of the roll rate during the manoeuvre, as shown in Fig. 8. Also, based on a theoretical understanding of the model dynamics (Equation (15)), for a rudder input, the derivatives $n_{v}, n_{r}$ and $n_{\zeta}$ were expected to be more significant than $n_{p}$.

In Step 3 of the process, all parameters again had sensitivities below the $10 \%$ threshold but the standard errors for $y_{v}, y_{p}$ and $n$, remained greater than $20 \%$. The derivative with the largest standard

Table 7

Results for maximum likelihood analysis of the yaw dynamics Steps 1 and 2

\begin{tabular}{|c|c|c|c|c|c|c|c|c|}
\hline & & Step 1 & & & & tep 2 & & \\
\hline & $\hat{\theta}$ & $\bar{s}$ & $\bar{I}$ & $\hat{\theta}$ & $\bar{s}$ & $\bar{I}$ & $\rho_{n p}$ & $\bar{\Theta}_{n p}$ \\
\hline$y_{v}$ & -0.178 & $69 \cdot 12$ & $8 \cdot 13$ & -0.245 & 38.67 & 5.96 & $0 \cdot 15$ & -0.09 \\
\hline$y_{p}$ & $0 \cdot 683$ & $59 \cdot 01$ & $5 \cdot 62$ & 0.950 & $28 \cdot 13$ & $4 \cdot 10$ & $0 \cdot 20$ & $0 \cdot 12$ \\
\hline$y_{r}$ & $-29 \cdot 29$ & $1 \cdot 15$ & $0 \cdot 13$ & $-29 \cdot 47$ & $0 \cdot 64$ & $0 \cdot 14$ & $0 \cdot 09$ & -0.04 \\
\hline$y_{\zeta}$ & $0 \cdot 212$ & $170 \cdot 3$ & $21 \cdot 62$ & $0^{*}$ & - & - & - & - \\
\hline$n_{v}$ & $3 \cdot 644$ & $7 \cdot 66$ & 0.90 & $3 \cdot 636$ & $7 \cdot 49$ & $0 \cdot 90$ & $0 \cdot 81$ & -0.60 \\
\hline$n_{p}$ & $1 \cdot 248$ & $79 \cdot 76$ & $6 \cdot 99$ & $1 \cdot 263$ & $76 \cdot 88$ & $6 \cdot 91$ & 1.00 & $1 \cdot .00$ \\
\hline$n_{p}$ & $-3 \cdot 273$ & $26 \cdot 84$ & 2.73 & $-3 \cdot 262$ & $25 \cdot 80$ & $2 \cdot 74$ & $0 \cdot 82$ & -0.70 \\
\hline$n_{\zeta}$ & $-5 \cdot 816$ & $15 \cdot 50$ & 1.79 & $-5 \cdot 806$ & $14 \cdot 74$ & 1.79 & $0 \cdot 78$ & -0.58 \\
\hline$\tau_{\zeta}$ & $0 \cdot 282$ & $1 \cdot 28$ & 0.48 & $0 \cdot 281$ & $2 \cdot 66$ & $0 \cdot 50$ & 0.42 & $0 \cdot 20$ \\
\hline
\end{tabular}

* - Removed from model structure

Table 8

Results for maximum likelihood analysis of the yaw dynamics Steps 3-5

\begin{tabular}{|c|c|c|c|c|c|c|c|c|c|c|}
\hline$\hat{\theta}$ & $\bar{s}$ & Step 3 & $\rho_{y v}$ & $\bar{\Theta}_{y v}$ & $\hat{\theta}$ & Step 4 & $\bar{I}$ & $\hat{\theta}$ & Step 5 & $\bar{I}$ \\
\hline$-0 \cdot 215$ & $43 \cdot 83$ & 5.96 & $0 \cdot 15$ & -0.09 & $0^{*}$ & - & - & $0^{*}$ & - & - \\
\hline 0.831 & $31 \cdot 72$ & $4 \cdot 10$ & $0 \cdot 20$ & $0 \cdot 12$ & $0 \cdot 374$ & $55 \cdot 01$ & 11.95 & $0^{*}$ & - & - \\
\hline $0^{*}$ & - & - & - & - & $0 *$ & - & - & $0^{*}$ & - & - \\
\hline $3 \cdot 918$ & $4 \cdot 21$ & 0.90 & $0 \cdot 81$ & -0.60 & 4.008 & 3.98 & 0.83 & $3 \cdot 981$ & 3.92 & 0.82 \\
\hline $0^{*}$ & - & - & - & - & $0 *$ & - & - & $0^{*}$ & - & - \\
\hline $0 \cdot 276$ & 3.03 & 0.50 & 0.42 & $0 \cdot 20$ & 0.278 & 1.78 & 0.57 & $0 \cdot 284$ & $2 \cdot 55$ & 0.53 \\
\hline
\end{tabular}

* - Removed from model structure 

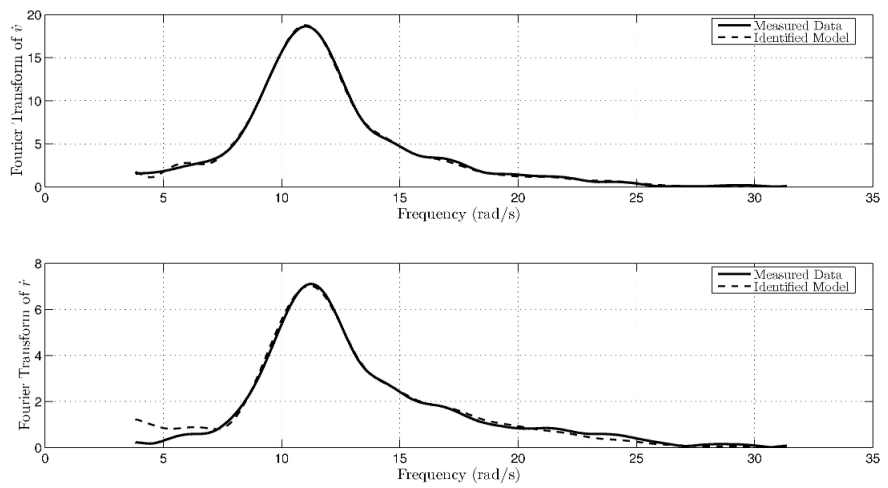

Figure 11. Comparison of measured and identified yaw responses in the frequency domain.

error was $y_{\nu}$ so the correlation between this parameter and the other derivatives was examined, with the correlation coefficients and confidence ellipsoid elements given in the last two columns of the first section of Table 8. Both metrics indicated that some correlation existed between $y_{v}$ and $y_{p}$ and it was decided that, based on the theoretical model in Equation (15), $y_{v}$ would be removed from the equations of motion. When this was carried out, the next run of the parameter estimation algorithm revealed $y_{p}$ to have an insensitivity of $11.95 \%$. While this value was close to the $10 \%$ guideline, it was much larger in comparison to the other insensitivities. The standard error for $y_{p}$ was also large at $55 \%$. Therefore, $y_{p}$ was also dropped from the model.

When $y_{p}$ was removed from the equations of motion and parameter estimation was repeated, all insensitivities and standard errors fell within the thresholds, as shown in the last section of Table 8. Therefore, the process was halted at this point. Figure 11 compares the identified response from the final model with the measured data in the frequency domain.

As with the UAV flight test data example in Section 4, the final model structure from maximum likelihood analysis matched that from stepwise regression, further validating the chosen threshold values for the statistical metrics. There was also close agreement between the final parameter estimate values from each method, as shown in Tables 4, 6 and 8. Figure 12 shows a comparison of the time histories of lateral acceleration and yaw acceleration identified using the stepwise regression and maximum likelihood techniques. A close match between the two identified models and the measured response was obtained.

The two examples presented in this paper indicate that the model structure determination process is a helpful tool for determining the most appropriate aerodynamic model. However, the examples also illustrate that the process works best when used in conjunction with an understanding of the aircraft dynamics, particularly when making borderline decisions on whether terms should be added or removed from the aerodynamic model. It is also important to note that, as already mentioned in Section 4, model structure determination will only identify as significant derivatives for which enough information exists within the data to reliably estimate the term. The fact that a derivative is not identified as important does not necessarily indicate that the parameter is insignificant for the configuration being analysed. To reach a conclusion, the analyst must decide whether the manoeuvre flown or control surface inputs applied were appropriate for exciting the derivative of interest. For example, for the wind tunnel data presented here, the parameter $n_{p}$ was considered to be insignificant. However, combining the rudder doublet data with that from an appropriate aileron input may lead to more information about $n_{p}$, allowing a statistically reliable estimate of the derivative to be obtained. It could possibly be argued that the doublet was not the most appropriate input to use for analysing cross-coupling effects. It
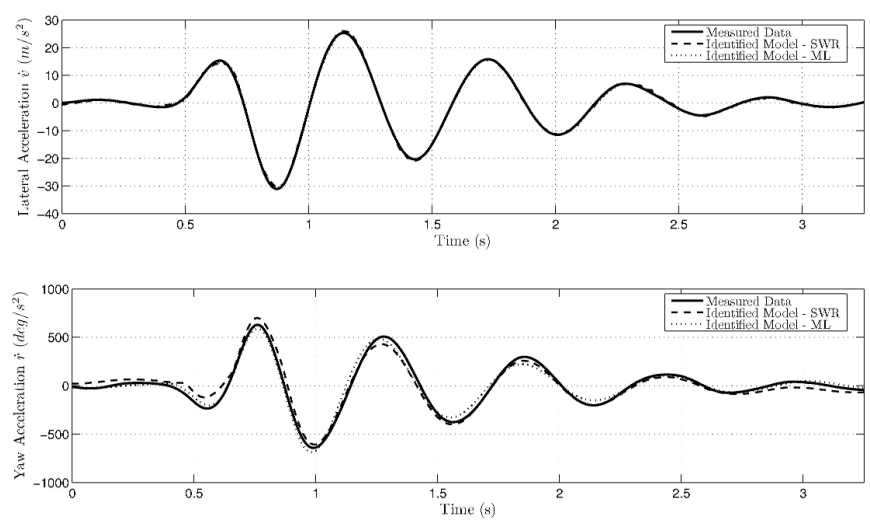

Figure 12. Comparison of lateral and yaw acceleration time histories from stepwise regression and maximum likelihood analysis.

excites a relatively narrow band of frequencies in comparison to other system identification manoeuvres, such as 3-2-1-1 inputs or frequency sweeps ${ }^{(1,2)}$. Also, in the free response of the aircraft (once the doublet has been completed and the control surface has returned to the neutral position), the motion variables tend to 'lock' together i.e. they are correlated. Inputs such as frequency sweeps generally 'unlock' the motion variables to play their own role. Therefore, it could be that more information about $n_{p}$ would have been obtained if a different input, such as a frequency sweep, had been used instead of a doublet. The above issue may be crucial for unconventional configurations exhibiting more significant cross-coupling effects and, where possible, it should be ensured that the control surface deflections applied are optimal for exciting such cross-coupling of the aircraft dynamics.

\subsection{CONCLUSIONS}

This paper has outlined the process of aerodynamic model structure determination from experimental data and examples of its application to UAV data have been presented. The process can be useful for determining the aerodynamic model of aircraft with unconventional configurations, which some UAVs tend to have. Two model structure determination techniques were described. The first was the well-established stepwise regression method, while the second was an adaptation of a frequency response method which uses estimates of the parameter standard deviations, parameter insensitivity and correlation among parameters to assess the adequacy of the aerodynamic model. However, the estimation process was carried out using maximum likelihood estimation rather than the frequency response approach. The principal advantage time domain maximum likelihood estimation has over frequency response identification is that nonlinearities can be estimated directly. Stepwise regression and maximum likelihood analysis were then performed on two data sources. The first was a set of UAV flight test data and the second was data recorded from dynamic wind-tunnel tests on a UAV configuration. For both examples, the final aerodynamic model determined using the maximum likelihood approach matched that found from stepwise regression, indicating that the threshold values selected for the maximum likelihood statistical metrics were reliable. However, it was noted that the examples presented in this paper involved relatively linear aircraft dynamics and relatively conventional airframe configurations. These examples have been used to help validate that maximum likelihood approach for simpler linear problems, before assessing whether the technique is reliable for more complex nonlinear data. This is the focus of further research. It is clear that the model structure determination process cannot be 
used blindly but requires engineering judgement, particularly when making borderline decisions on whether terms should be added or removed from the aerodynamic model. It is a tool that is most helpful when used in conjunction with an understanding of the aircraft physics. Further, model structure determination will only identify as significant derivatives for which enough information exists within the data to reliably estimate the term. It is crucial to ensure that the control surface deflections applied during the flight test are suitable for exciting the dynamics of interest.

\section{ACKNOWLEDGEMENTS}

The authors wish to thank the EPSRC and BAE Systems for funding this research. In particular, thanks go to Chris Fielding from BAE Systems for his continued support and providing feedback on the contents of the paper.

\section{REFERENCES}

1. Klein, V. and Morelli, E.A. Aircraft system identification: Theory and practice, 2006, American Institute of Aeronautics and Astronautics, Reston, VA, USA.

2. JATEGAONKAR, R.V. Flight vehicle system identification: A time domain methodology, 2006, American Institute of Aeronautics and Astronautics, Reston, VA, USA

3. TisChLER, M.B. and REMPLE, R.K. Aircraft and rotorcraft system identification: Engineering methods with flight test examples, 2006, American Institute of Aeronautics and Astronautics, Reston, VA, USA.

4. KLEIN, V. On the adequate model for aircraft parameter estimation, 1975, College of Aeronautics Aero Report 28, Cranfield Institute of Technology, UK.

5. TAYLOR, L.W. Application of new criterion for modeling systems, May 1975, AGARD CP-172, 4.1-4.9.

6. Hall, W.E., GuPTA, N.K. and Tyler, J.S. Model structure determination and parameter identification for nonlinear aerodynamic flight regimes, May 1975, AGARD CP-172, 21.1-21.21.

7. Gupta, N.K., Hall, W.E. and Trankle, T.L. Advanced methods of model structure determination from test data, $J$ Guidance, Control and Dynamics, 1978, 1, (3), pp 197-204.

8. Klein, V., Batterson, J.G. and Murphy, P.C. Determination of airplane model structure from flight data using modified stepwise regression, October 1981, NASA TP-1916.
9. Klein, V. and Batterson, J.G. Determination of airplane model structure from flight data using splines and stepwise regression, March 1983, NASA TP-2126.

10. MiLne, G.W. Identification of a Dynamic Model of a Helicopter from Flight Tests, 1986, PhD thesis, Stanford University, Department of Aeronautics and Astronautics, CA, USA

11. Carnduff, S. System Identification of Unmanned Aerial Vehicles, 2008, PhD thesis, School of Engineering, Cranfield University, UK.

12. FLUX, P. Some stability and control aspects of UCAV configurations, 2003, CEAS Aerospace Aerodynamics Research Conference, June 2003, London, UK.

13. Montgomery, D.C., Peck, E.A. and Vining, G.G. Introduction to Linear Regression Analysis, 2001, Wiley, New York, USA.

14. KreysZig, E. Advanced Engineering Mathematics, 1999, Eighth edition, John Wiley, New York, USA.

15. Tischler, M.B. and KaletKa, J. Modeling XV-15 tilt-rotor aircraft dynamics by frequency and time-domain identification techniques, December 1986, NASA-TM-89404.

16. IlifF, K.W. and TAYLOR, L.W. Determination of stability derivatives from flight data using a Newton-Raphson minimization technique, 1972, NASA TN D-6579.

17. Maine, R.E. and ILIFF, K.W. The theory and practice of estimating the accuracy of dynamic flight-determined coefficients, 1981, NASA Reference Publication 1077.

18. Morelli, E.A. and Klein, V. Accuracy of aerodynamic model parameters estimated from flight test data, $J$ Guidance, Control and Dynamics, 1997, 20, (1), pp 74-80.

19. ESDU Aerodynamics Series, Engineering Sciences Data Unit, ESDU International, London, UK.

20. USAF stability and control DATCOM, Flight Control Division, US Air Force Flight Dynamics Laboratory, Wright-Patterson Air Force Base, Fairborn, OH, USA.

21. Carnduff, S.D., Erbsloeh, S.D., Cooke, A.K. and CooK, M.V. Characterizing stability and control of subscale aircraft from windtunnel dynamic motion, J Aircr, 2009, 46, (1), pp 137-147.

22. MCDougall, N. BAE Systems/EPSRC Integrated research Programme in aeronautical engineering, Aeronaut J, 2006, 110, (1104), pp 121-124.

23. CARndufF, S.D. and CoOKe, A.K. Formulation and system identification of the equations of motion for a dynamic wind tunnel facility, March 2008, College of Aeronautic Report 0801, Cranfield University, UK

24. Cook, M.V. Flight Dynamics Principles, 1997, Edward Arnold, London, $\mathrm{UK}$.

25. Marchand, M. and Fu, K.H. Frequency domain parameter estimation of aeronautical systems without and with time delays, 1985, proceedings of 7th IFAC Symposium on Identification and System Parameter Estimation, July 1985, pp 669-674, Pergamon, Oxford, UK 